Francisco Caamaño, Discípulos y disidentes: la formación de las revistas político-culturales como espacios de ruptura frente al Partido Comunista argentino (1959-1969), Izquierdas, 49, julio 2020:1018-1035

\title{
Discípulos y disidentes: la formación de las revistas político-culturales como espacios de ruptura frente al Partido Comunista argentino (1959-1969)
}

Disciples and dissidents: the formation of political-cultural magazines as spaces of rupture against the Argentine Communist Party (1959-1969)

\author{
Francisco Caamaño*
}

Resumen: El propósito de este artículo es discutir sobre la trayectoria de cuatro colectivos culturales de la izquierda argentina, disidentes del Partido Comunista local, que a lo largo de la década de 1960 formaron diversas revistas como modo de expresar esa ruptura. A partir del análisis del discurso de estos grupos y la utilización de algunas categorías temporales benjaminianas, se buscará encontrar las posibles causas por las cuales estos litigios con el partido se cristalizaron en esta vía singular.

Palabras claves: Nueva izquierda argentina, Partido Comunista argentino, disidencias partidarias, revistas culturales.

\begin{abstract}
The aim of this article is to discuss the trajectory of four cultural collectives of the Argentine Left, dissidents of the local Communist Party, which during the 1960s produced several magazines as the way to express that rupture. From the analysis of the discourse of these groups and the use of some Benjaminian temporal categories, it will be looked forward to find the possible causes for which these litigations against the party crystalized in this particular way.
\end{abstract}

Key words: New Argentine Left, argentinian Communist Party, party dissidence, cultural magazines.

Recibido: 1 abril 2019 Aceptado: 22 mayo 2019

\footnotetext{
* Argentino. Joven investigador de la Universidad Nacional de La Plata. Miembro del proyecto de investigación "Los sentidos del pasado: historiografías, prácticas culturales, memorias" con procedencia de la misma institución (código H824). Este artículo se realizó en el marco de la beca "Estímulo a las Vocaciones Científicas" del Consejo Interuniversitario Nacional (CIN), centrado en las revistas culturales de la izquierda argentina durante la década de 1960. Correo electrónico: franciscocaama@gmail.com
} 
Francisco Caamaño, Discípulos y disidentes: la formación de las revistas político-culturales como espacios de ruptura frente al Partido Comunista argentino (1959-1969), Izquierdas, 49, julio 2020:1018-1035

\begin{abstract}
Los buenos, ciertamente, no pueden crear: son siempre el comienzo del fin: crucifican a quien escribe nuevos valores sobre nuevas tablas, sacrifican el futuro ante el altar de sí mismos, jcrucifican todo el porvenir de los hombres! Friedrich Nietzsche, Así habló Zaratustra. Un libro para todos y para nadie, 1885 .
\end{abstract}

\title{
Introducción
}

Desde los albores de la Revolución Rusa hasta los años próximos a nuestra historia contemporánea, la cultura comunista en la Argentina constituyó un ideario esencial dentro del pensamiento clásico de la izquierda nacional. Si bien aún subsisten algunos debates en torno a su carácter -o no- de movimiento de masas, la certeza es que, pese a su relativa marginalidad, la identidad comunista logró a lo largo de sus años de existencia interpelar a numerosos actores sociales. En términos gramscianos, el comunismo como ideología desempeñó un papel de orientación social, organizando a diferentes grupos humanos dentro del espacio en el cual estos accionaban, ya sea con la finalidad de la preservación o transformación de su realidad.

Con un fuerte atractivo para la clase obrera durante las décadas de 1920 y 19301, la hegemonía peronista sobre el movimiento obrero eclipsó el papel del comunismo dentro de los trabajadores, dando a este ideario social un perfil más ligado a capas medias ilustradas e intelectuales ${ }^{2}$. Con un catastrófico declive a principios de la década de 1990, la cultura comunista en Argentina comenzó a ser cada vez más rigurosamente un objeto de análisis por parte de numerosos trabajos. Precedidos por una serie de escritos de tipo partidario, encargados de reconstruir biográficamente la historia del Partido Comunista argentino (PCA), los estudios historiográficos que abordan la temática del campo comunista nacional son un aspecto naciente pero diversamente nutrido ${ }^{3}{ }^{4}$. En la actualidad, las indagaciones de la historiadora Adriana Petra ${ }^{5}$ son las producciones más elaboradas de esta problemática. Como comenta la investigadora, los estudios sobre el comunismo en Argentina están nucleados en torno a la organización madre, es decir, el PCA, aunque no limitados al mismo. Efectivamente, el análisis de los vínculos entre partido y otros sectores sociales, a través de los variados artefactos culturales elaborados a lo largo de todo el siglo XX, toma una dimensión central al momento de concentrarse en los casos de las rupturas y disidencias del PCA. Este problema será uno de los ejes centrales de este artículo.

Adentrándonos en los años pioneros de la década de 1960, el PCA fue víctima de una serie de relevantes resquebrajamientos en el seno de su organización. Simultáneamente al declive del estado soviético como eje vertebrador y hegemónico dentro del campo del socialismo internacional, el PCA sufrió una multiplicación y explicitación de variadas divergencias y rupturas ${ }^{6}$. Convertidas algunas de ellas en otras organizaciones políticas, como es el arquetípico caso del Partido Comunista Revolucionario 7 , otras disidencias se expresaron concretamente en la formación de revistas político-culturales. De esta

\footnotetext{
${ }^{1}$ Camarero Hernán, Tiempos rojos. El impacto de la Revolución Rusa en la Argentina, Buenos Aires, Sudamericana, 2017.

2 Campione Daniel, El Partido Comunista de la Argentina. Apuntes sobre su trayectoria, Coloquio internacional: El comunismo. Otras miradas desde América Latina, México D.F, 2007.

${ }^{3}$ Un trabajo pionero en este sentido es Falcón Ricardo \& Quiroga Hugo, Contribución al estudio de la evolución ideológica del PCA, En Contribuciones programa CLACSO-Santiago de Chile, 50, 1987.

${ }_{4}$ Para un análisis de carácter historiográfico, ver Cernadas Jorge, Pittaluga Roberto \& Tarcus Horacio, La historiografía sobre el Partido Comunista de la Argentina: Un estado de la cuestión, En El Rodaballo. Revista de política y cultura, 8, 1998.

5 Petra Adriana, Intelectuales y cultura comunista: Itinerarios, problemas y debates en la Argentina de posguerra, Buenos Aires, Fondo de cultura económica, 2017.

${ }^{6}$ Las rupturas y disidencias sufridas por el PCA no son una cuestión aislada y propia de la tradición comunista. Observando el fenómeno desde un enfoque nacional, podemos considerar que esta crisis es común a todos los partidos tradicionales de la izquierda argentina. Para un abordaje profundo de la temática, ver Tortti María Cristina, El "viejo" Partido Socialista y los orígenes de la "nueva" izquierda (1955-1965), Buenos Aires, Prometeo libros, 2009.

7 Siskindovich Santiago, El Partido Comunista Revolucionario y la construcción de la delimitación con el Partido Comunista argentino (19681969), En Izquierdas, 49, enero 2020, 43-57.
} 
forma, numerosos militantes y simpatizantes del partido, provenientes primordialmente de los círculos juveniles, propulsaron la formación de grupos culturales alternativos que cuestionaban los valores políticos y estéticos difundidos por el partido madre. De estas experiencias, nacieron las revistas Pasado y Presente (PyP), La Rosa Blindada (RB), El Grillo de Papel (EGDP) y El Escarabajo de Oro (EEDO), ejemplos emblemáticos de esta nueva generación ${ }^{8}$. A partir del estudio comparativo de estos cuatro emprendimientos culturales, se intentará establecer algunos criterios que nos permitan visualizar dichas experiencias como un todo articulado. Si bien estos artefactos contienen profundas diferencias, se buscará demostrar la existencia de ciertos aspectos que nos permitan unificar a estos agrupamientos de un modo elocuente.

En ese marco, en el presente escrito se tomarán dos temas centrales a indagar. En primer lugar, se profundizará sobre el rol de las revistas como herramientas de disidencia, utilizadas éstas por distintos grupos intelectuales para la formulación de una identidad y personalidad singular, distinguible de la antigua organización que previamente definía su lugar en el campo social. De este modo, la pregunta central a realizar está vinculada con la otra vía de ruptura observada en estos casos: el partido político. ¿Por qué esos colectivos se agruparon alrededor de una revista? ¿Por qué no formaron una estructura partidaria alternativa? En segundo lugar, se recreará una disputa ideológica bastante común dentro del campo de la izquierda política: lo que Horacio Tarcus denomina la "querella del marxismo"9. Propio de la tradición del materialismo histórico contemporáneo, la contienda entre los diversos lectores de Marx en torno a una "correcta" interpretación de los legados del genio del Tréveris es una moneda corriente a lo largo de todo el siglo XX. Las luchas entre las diversas formas de recepcionar los escritos del intelectual alemán nos permiten definir al marxismo como un corpus ideológico complejo y multifacético en su interior, el cual no finaliza en la mera lectura del propio Marx. En ese marco, observamos que en todas las revistas estudiadas se encuentra la reivindicación de un marxismo crítico, humanista y heterodoxo frente a otro que, representado por los cuadros del PCA, es caracterizado como "ortodoxo", "vulgar" y "monolítico" y subordinado totalmente a los objetivos de supervivencia de la Unión Soviética. Este elemento, primordial para unificar todas estas experiencias abigarradas, se observa constantemente en los artículos, secciones culturales y notas de opinión de todas las revistas, dentro de las cuales aparecen polémicas abiertas con otros artefactos culturales -como las revistas Cuadernos de Cultura y Gaceta Literariaque son consideradas defensoras de los posicionamientos partidarios del comunismo argentino. En síntesis, en este trabajo no solo se busca referir a un problema de la historia cultural -las revistas como artefacto cultural-, sino que, además, se busca ampliar el contenido y abarcar la discusión sobre el marxismo occidental, entendido ello como un problema propio de la historia intelectual de las izquierdas.

Con dichos objetivos en mente, este artículo está dividido en tres apartados. El primero de ellos introduce brevemente la formación de la nueva izquierda intelectual argentina, priorizando la descripción del contexto social e intelectual dentro del cual esta novedosa intelectualidad emergió, a la vez que se profundizará en el sentido que para estos grupos tuvo la utilización de las revistas políticas. En el segundo apartado, se reconstruirán los procesos de rupturas de los tres colectivos culturales seleccionados frente al PCA, detallando cómo se dieron estos procesos y manifestando cómo estas separaciones se observan en las revistas. Se describirá a cada uno de los colectivos de manera separada y aislada, pero marcando constantemente ejes conectivos entre todas las experiencias. La selección del orden de presentación de cada grupo no responde a un criterio cronológico, sino que se pensó en relación al grado de importancia

8 Sobre PyP, ver Petra Adriana, Pasado y Presente: Marxismo y modernización cultural en la Argentina postperonista, En Historia y espacio, 41, 2013; y Massholder Alexia, El surgimiento de la revista Pasado y Presente. Una mirada desde lo generacional, Jornadas Internacionales José María Aricó, Córdoba, 2011. Para RB, ver Kohan Néstor, Cultura y revolución en La Rosa Blindada, Buenos Aires, Amauta Insurgente ediciones, 2015; y Katchadjian Guillermo, La Rosa Blindada: entre la cultura de la rebelión y la politica revolucionaria, IV Jornadas de Jóvenes Investigadores. Instituto de Investigaciones Gino Germani, Facultad de Ciencias Sociales, Universidad de Buenos Aires, Buenos Aires, 2007. Para EGDP y EEDO, ver Grande Cobián, Leonardo J., El programa político de El Grillo de Papely El Escarabajo de Oro (1959-1964), IV Jornadas de Sociología de la UNLP, La Plata, 2005.

9 Tarcus Horacio, Marx en Argentina. Sus primeros lectores obreros, intelectuales y cientificos, Buenos Aires, Siglo XXI, 2007. 
que representó para el PCA la ruptura de cada grupo estudiado. Dada la influencia y el carácter casi mediático que tuvo PyP, esta revista será estudiada primero, seguida por RB y concluyendo con el EGDP y EEDO, revistas que nucleaban al grupo de Abelardo Castillo que, como se explicitará más adelante, no pertenecían íntegramente a la estructura partidaria del PCA. Por último, este trabajo abordará las posibles causas de la no formación de un partido político por parte de estos colectivos como forma de expresar la disidencia, para lo cual se utilizará para la explicación algunas categorías temporales de Walter Benjamin que, se cree, pueden mantener una relación con los discursos de las revistas estudiadas.

\section{Las revistas y la autonomización intelectual: el nacimiento de una nueva izquierda en la Argentina}

El número trece de la revista El Escarabajo de Oro, publicada en el mes de abril de 1962, aporta como novedad la utilización de un nuevo epíteto que acompaña la presentación de la portada: Di tu palabra, y rómpete. La cita, extraída - de manera incompleta- de la obra de Friedrich Nietzsche ${ }^{10}$, no es la primera frase que decora la tapa de esta revista. ${ }^{11}$ Pese a su aparente trivialidad, la frase expresa de manera sintomática la personalidad de toda una nueva generación de intelectuales de izquierda. La palabra -escrita- no es una mera abstracción: es la vía concreta de accionar dentro de su espacio social; es la consumación de la identidad del intelectual, su sentido ser y esencia. De allí se entiende la reproducción de la trágica sentencia a Zaratustra: expresa tu palabra, afirma tu legado, y posteriormente, destrúyete "rómpete, en pedazos". No hay camino más relevante en la configuración de un sujeto de letras. No hay nada más imprescindible que manifestar la - "su"- palabra. Es su realización y plenitud: lo demás, nada tiene importancia.

De allí se entiende el lugar primordial que puede ocupar una revista para un colectivo intelectual. Obra de corto aliento, con una facultad de intervención rápida y eficaz, la revista es un artefacto cultural que supone la difusión de la palabra en una dimensión pública. No obstante, la revista no es un elemento constante e indeterminado. Su empleo y difusión atraviesa distintos periodos, momentos en donde sus números estallan cuantitativa y cualitativamente, e instantes de eclipses, de poca utilización de esta herramienta cultural. Durante la década de 1960 en Argentina, nos encontramos con una explosión significativa de emprendimientos culturales alternativos, primordialmente revistas de crítica política y estética. Desde la óptica de la historiografía local, este fenómeno responde a la formación de una "nueva izquierda intelectual", categoría que alude a la recreación de una inédita figura intelectual que actúa como "antecedente significativo -o tramo inicial- del proceso de politización desatado a partir del Cordobazo"12. Reflejados en la imagen del compromiso sartreano, esta joven vanguardia empleó a la revista como medio central para difundir sus perspectivas. Abandonando las concepciones universales del pensador antifascista, defensor de los valores culturales liberales y progresistas, la nueva intelectualidad repensó el aspecto nacional desde variadas ópticas, tomando al movimiento peronista como un eje central de sus preocupaciones. En un mundo extremadamente convulsionado por acontecimientos como la Revolución Cubana o la Guerra de Argelia, este naciente paradigma de intelectual de izquierdas revalorizó los ya no ajenos movimientos tercermundistas, como complemento o incluso sustituto de potenciales vías

${ }^{10}$ Con respecto a esto, cabe destacar que dicha cita forma parte íntegra de la tradición de las revistas argentinas y latinoamericanas. Su mención data de fechas pretéritas a la formación de El Escarabajo de Oro. Hacia la década de 1920, la encontramos en las revistas Verbo Libre y la universitaria Insurrexit -ambas del año 1920- y más adelante, en la peruana Claridad-del año 1922-.

11 Inicialmente aparece la clásica cita que Goethe emplea en su célebre Fausto: "Gris es toda teoría y verde el árbol de oro de la vida". Durante una breve etapa del año 1970, la revista fue encabezada por un dicho de Oscar Wilde: "Nada de lo que actualmente sucede tiene la menor importancia”.

12 Tortti María Cristina, Protesta social y "Nueva Izquierda" en la Argentina del "Gran Acuerdo Nacional", Taller. Revista de Sociedad, Cultura y Política, 6, 1998. 
emancipadoras, como es el caso del modelo soviético ${ }^{13}{ }^{14}$. Cabe agregar, la nueva intelectualidad de izquierda no es la única heredera de la tradición antifascista de la década de 1930. Simultáneamente, los inicios de los años '60 son testigos del funcionamiento de otra corriente de pensadores cuyo origen se remonta a los primeros años de la década de 1950. Me refiero aquí a los liberales antitotalitarios, intelectuales agrupados en torno a los principios del Congreso por la Libertad de la Cultura. Desde otra tónica, este extenso grupo compartió con la nueva izquierda intelectual su crítica al comunismo soviético, representado localmente por el PCA. No obstante, la oposición a lo soviético fue el sentido ser de este colectivo, que abocó sus actividades a ser un contrapunto de la política cultural soviética. Integrando un movimiento que accionaba a escala global, los miembros del Congreso por la Libertad de la Cultura se presentaban como leales defensores de la democracia liberal occidental, es decir, como atentos rivales de cualquier forma de totalitarismo, tanto de derecha como de izquierda. Sus filas estaban compuestas por prestigiosos intelectuales como Victoria Ocampo, Francisco Romero, Jorge Luis Borges, Ernesto Sábato, Claudio Sánchez Albornoz, Alfredo Palacios, Nicolás Repetto, entre otros, algunos quienes habían participado de las movilizaciones antifascistas durante las décadas de 1930 y 1940.15

Volviendo a la presentación de la nueva intelectualidad de izquierda, aparece presente dentro de estos flamantes pensadores un proceso de "autonomización" de los mismos respecto a las estructuras partidarias tradicionales, como el propio PCA o el Partido Socialista Argentino. La convergencia de una serie de sucesos a escala global y local a lo largo de varias décadas catalizará una crisis dentro de la identidad comunista de gran parte de las capas medias ilustradas a nivel mundial. Dicho proceso, cuyo comienzo se asocia al disruptivo XX Congreso del Partido Comunista de la Unión Soviética de 1956, tiene diversas instancias particulares en los itinerarios personales de cada sujeto. Desde el punto de vista de Silvina Sigal, durante la década de 1960 la intelectualidad crítica entra en una fase de "puesta en disponibilidad ideológica como consecuencia de la ruptura de lealtades y commitments anteriores."16, fenómeno dentro del cual la juventud comunista no estuvo exenta. No obstante, estos desarrollos son indicativos, pero no absolutos para explicar el desenlace de estos procesos culturales. La conjunción de núcleos intelectuales - previamente ligados al PCA, ya sea como militantes orgánicos o meros simpatizantes- en torno a revistas alternativas es un aspecto que debe ser analizado dentro de la propia biografía de cada uno de estos grupos, sin remitir exclusivamente a los fenómenos más globales o estructurales. Como afirma Fernanda Beigel, "no hay relación concéntrica entre el texto y el contexto", siendo poco productivo explicar y equiparar estos discursos colectivos a procesos generales ${ }^{17}$. En ese marco, en este trabajo se procederá a describir la formación de cuatro emprendimientos culturales, los cuales pese a sus diferencias y matices reúnen un conjunto de atributos que permiten pensarlos como experiencias comunes. La temporalidad de análisis seleccionada -la etapa que comprende desde el año 1959 hasta el año 1969- no remite al periodo de existencia de las revistas elegidas; de hecho, El Escarabajo de Oro mantuvo activos sus números hasta el año 1974. Más bien, el recorte temporal responde a varias razones. En primer lugar, es entonces donde la disputa con el PCA se manifiesta más visiblemente en las revistas. En segundo lugar, esta es la etapa en la cual se observa más claramente el perfil humanista de estos emprendimientos culturales -el coqueteo con el estructuralismo se observará más adelante-; y, por último, esta cronología coincide parcialmente con la etapa en la cual la historiografía engloba el florecimiento de la nueva izquierda intelectual en Argentina. Enmarcadas dentro de la tradición comunista, pero intensamente escindidas de la estructura partidaria, estas revistas actuaron como un

\footnotetext{
13 Terán Oscar, Nuestros años sesenta. La formación de la nueva izquierda intelectual argentina (1956-1966), Buenos Aires, Puntosur editores, 1991.

14 Gilman Claudia, Entre la plumay el fusil. Debates y dilemas del escritor revolucionario en América Latina, Buenos Aires, Siglo XXI, 2003. 15 Nállim, Jorge A., Intelectuales y Guerra Fría: El Congreso por la Libertad de la Cultura

en Argentina y Chile, 1950-1964. En Anuario del Instituto de Historia Argentina, 14, 2014.

16 Sigal Silvia, Intelectuales y poder en la década del setenta, Buenos Aires, Puntosur editores, 1991.

17 Beigel Fernanda, Las revistas culturales como documentos de la bistoria latinoamericana, En Utopía y Praxis Latinoamericana, eneromarzo 2003, 105-115.
} 
punto de encuentro de itinerarios individuales en torno a un credo común. En síntesis, estas revistas fueron un espacio de enlace de un colectivo de pensadores, cuyo horizonte central es la defensa de un marxismo o una izquierda más libertaria al servicio de la emancipación social.

\section{La manifestación del parricidio. Disidencia y grupos culturales alternativos en el seno de la tradición comunista argentina}

Si observamos el recorrido de las revistas PyP, RB, EGDP y EEDO, visualizaremos dos aspectos primordiales. En primer lugar, una trayectoria similar: en todos los casos aparece un primer origen marcado por la cercanía a la cultura comunista partidaria, una posterior tensión y disputa con dicho espacio y finalmente, una explicitación de dicha diferencia. En segundo lugar, pese a ese itinerario relativamente análogo, se observan profundas diferencias de composición entre estos agrupamientos. Pasaremos a observar estas cuestiones en detalle.

Con una primera aparición entre los años 1963 y 1965, la revista Pasado y Presente constituye un hito central en la historia intelectual argentina. Impulsada por José María Aricó, Héctor Schmucler y Oscar Del Barco, este emprendimiento cultural es catalogado como el más significativo de esta nueva generación, reiteradamente mencionado y aludido, simulando actuar para la historiografía como un tipo ideal weberiano de la nueva izquierda intelectual nacional. En sus páginas, la revista lleva impresa la historia de un colectivo de jóvenes talentosos del PCA, quienes buscan revitalizar al marxismo frente a una estructura partidaria que se les presentaba como completamente anacrónica. Integrada en gran parte por intelectuales que atravesaron el ámbito universitario, el grupo pasadopresentista apostó por una integración de la tradición marxista con los conocimientos académicos del campo de las Ciencias Sociales, con el íntegro objetivo de contribuir al proceso revolucionario en la Argentina. Pese a negar poseer algún tipo de maestro, parte del grupo fue formado teóricamente por una reconocida figura partidaria como Héctor P. Agosti. Financiada inicialmente por el propio PCA, la manifestación de una lealtad menguante con respecto al partido en su primer número de abril de 1963 produjo la expulsión de los integrantes de la revista de la célula partidaria. Dicho devenir no fue una sorpresa para los autodenominados "jóvenes gramscianos", cuyos integrantes nucleados en Córdoba continuaron con el emprendimiento de manera autónoma. A excepción de unas breves tentativas de Portantiero de fundar la organización Vanguardia Revolucionaria, los marginados discípulos de Agosti tomaron a la redacción de la revista como su principal tarea política luego de su polémica exclusión del partido.

Similarmente, el pionero colectivo editorial de La Rosa Blindada también se formó íntegramente en el interior del campo cultural comunista. Sus dos principales impulsores, Carlos Alberto Brocato y José Luis Mangieri, eran militantes del PCA. De hecho, hasta el sexto número de la revista, el poeta comunista Raúl González Tuñón fue el director honorífico de la misma. Como indica Néstor Kohan, el reconocimiento a uno de los intelectuales más consagrados dentro de la izquierda clásica manifiesta el estrecho vínculo que este colectivo mantuvo con sus maestros. Esta relación simbiótica con la tradición cultural comunista separa a esta revista -por lo menos, en términos discursivos- de la experiencia pasadopresentista ${ }^{18}$. Publicada entre los años 1964 y 1966, RB recreó una crítica al PCA en términos más bien estéticos que políticos. La expulsión de Brocato y Mangieri del partido, tras una polémica abierta con Agosti a través de su revista, fue completamente inesperada, distinto al caso de PyP. De esta forma, a partir de su quinto número, la revista abrió el espacio meramente artístico al debate político, integrando nuevas problemáticas que hasta el momento simulaban ser una simple tensión. Por su parte, las revistas El Grillo de Papel (1959-1960) y El Escarabajo de Oro (1961-1974) -y sin olvidar nombrar, la revista El Ornitorrinco- fueron pensadas por un mismo proyecto artístico. Contrariamente a las otras revistas mencionadas, estas entidades no fueron ideadas por militantes orgánicos del PCA. De hecho, estos

18 Kohan Néstor, La Rosa Blindada: una pasión de los sesentas, Buenos Aires, La Rosa Blindada, 1999. 
artefactos culturales fueron creados por antiguos miembros de Gaceta Literaria, editorial de tradición simpatizante pero no vasalla del PCA. Su director, Abelardo Castillo, se identificaba abiertamente con la herencia anarquista. Su cercanía inicial a Gaceta Literaria, emprendimiento dirigido por Pedro Orgambide, se debe a su admiración por la organización con que contaba la misma, siendo catalogada como una auténtica revista de literatura por nuestro protagonista. No obstante, la defensa de la clausura soviética a la obra de Boris Pasternak, tras la obtención del Premio Nobel de Literatura en 1958 ${ }^{19}$, marcó un primer paso en su separación definitiva con la publicación de Orgambide. Castillo logró acercar a su proyecto a parte de los integrantes de Gaceta Literaria, entre ellos, Arnoldo Liberman. La integración futura de Liliana Heker, joven y talentosa escritora, terminó de otorgar al grupo una silueta marcadamente apartidaria. A su modo, las revistas constituyeron un corpus literario en donde los problemas y discusiones estéticas eran vitales, en un grado mayor a PyP y RB. El debate público no era ajeno, y la publicación manifestaba sus consideraciones políticas a través de sus numerosos artículos. La relación con el PCA era ambigua: si bien admitía un rechazo a sus cánones ortodoxos, marcando distancia desde un lugar contestatario, en muchos casos la crítica era encubierta, tratando de flexibilizar la tensión con la organización partidaria. Como veremos, esta cuestión se explica por la estrategia política esgrimida por el grupo de Abelardo Castillo, el cual promulgaba una unidad de acción de amplio espectro dentro de la izquierda nacional.

Según la consideración de Agustín Cuevas, la descripción de la cultura comunista y sus entidades representantes -los partidos comunistas- como elementos ortodoxos, cerrados y dogmáticos no facilita una lectura correcta de dicha tradición. Tratando de matizar la visión que otorga una total dependencia pragmática de los partidos comunistas latinoamericanos con respecto a la Internacional Comunista, el autor intenta demostrar las visibles diferencias que existen entre las agrupaciones comunistas de todo el mundo $^{20}$. Pese a la validez teórica que pueda tener esta suposición, lo cierto es que, contrariamente al planteo de Cuevas, las revistas argentinas de la nueva izquierda intelectual percibían a su antagonista -el PCA- como una sociedad con dichas características. El uso por parte de estos agrupamientos de calificativos como "ortodoxos" y "dogmáticos" para referirse al PCA, es un aspecto recurrente y hasta cotidiano. En un contex to global en donde la conflictividad social llega a un grado profundo de ebullición, marcando la apertura a un periodo concebido como potencialmente revolucionario, la praxis de la Unión Soviética y sus representantes en las esferas nacionales parece pecar de conservadora.

Insertándonos en los discursos de todas las publicaciones analizadas, esta realidad no merece discusión. Aunque la disidencia puede tardar en explicitarse, la circunstancia amerita necesariamente una manifestación pública de las diferencias. En PyP y RB, la crítica se hará concreta y cotidiana tras la expulsión de sus redactores del PCA. Tras el exilio forzado de sus miembros, las revistas fueron puestas al servicio del debate, publicando un conjunto de artículos y editoriales que reprobaban las medidas ejercidas por el partido. El debate político no es un fin en sí mismo: es un punto de partida. La lucha contra el organismo madre implica un proceso de independencia, de madurez política: la necesidad urgente de conseguir una identidad propia mediante el ataque al adversario. Con este conflicto, ambos grupos se autodefinieron como otro colectivo cultural, pero, esencialmente, como otro marxismo posible. Una vez transcurrido el huracán de la disputa, la mención del antagonista ortodoxo disminuyó progresivamente. Restándole importancia, el dogmatismo soviético fue citado sin nombrar la sombra del PCA. En cambio, el grupo de Abelardo Castillo no nace al calor de una ruptura fuerte. Sus revistas,

\footnotetext{
19 Sobre esta situación, hay que tener en cuenta que Pasternak obtuvo el Premio Nobel contra su voluntad. Como figura en su correspondencia con Renata Schweitzer, cuando el escritor se enteró que había sido ternado para el Premio Nobel, escribió a la poeta comentándole la angustia que eso le provocaba, ya que nada podría empeorar tanto su situación como obtener, en ese momento, el Premio Nobel. Renata, ingenuamente, envió entonces una carta a la Academia Sueca solicitando que por favor postergasen un año la posible adjudicación a Pasternak del Premio Nobel -para así dar tiempo a que el escritor pudiese estar fuera de la URSS cuando se hiciese el anuncio-, y como justificativo de su pedido adjuntaba una copia de la carta que Pasternak le había enviado. A los pocos días, la Academia de Suecia, advertida, anunciaba a todo el mundo que Boris Pasternak era el ganador del Premio Nobel.

${ }^{20}$ Cuevas Agustín, El marxismo latinoamericano: historia y problemas actuales. En Entre la ira y la esperanza y otros ensayos de crítica latinoamericana, Bogotá, Siglo del Hombre Editores y CLACSO, 2008, 9-67.
} 
expresión escrita de su conciencia, no se afirman estrictamente como marxistas, sino que prefieren el adjetivo de "izquierda" o "socialista", a secas. Si bien la burla al marxismo soviético -y por extensión, al PCA- estuvo siempre presente, la seriedad y sistematización de la crítica se abrirá como un mecanismo de defensa frente a los ataques tanto de Gaceta Literaria cómo de los cuadros políticos del PCA.

"La tradición de todas las generaciones muertas oprime como una pesadilla el cerebro de los vivos" 21 . Esta tragedia, ratificada singularmente por Marx, parece sintetizar fantásticamente la réplica que los cordobeses gramscianos remarcan al PCA. Para PyP, la incapacidad que poseen los dirigentes de la estructura partidaria de romper con un corpus teórico raquítico condenaría al fracaso a todo el devenir emancipatorio en la Argentina. Su apego y reproducción cegada a los escritos y fórmulas teóricas del pasado impediría a los pregoneros comunistas accionar acorde a la realidad argentina. Según los integrantes de PyP, esto explicaría cómo los cuadros del PCA, herederos de una tradición formidablemente rica como el marxismo, no lograron conquistar la ansiada hegemonía dentro del proletariado nacional. En la mirada de estos jóvenes cordobeses, el problema reside, exclusivamente, en la cristalización de esa ideología liberadora, su superficial esquematización. Esta cuestión fue advertida por Aricó y Del Barco en el primer número de Pasado y Presente, aunque de una forma sutilmente implícita.

Enérgicamente separada del determinismo económico y el subjetivismo voluntarista, la política para el colectivo pasadopresentista implica una constante acción desmitificadora, la cual apela a la tradición del pensamiento marxista sin desmerecer el estudio de la situación concreta en la que tiene lugar la praxis transformadora. En su conceptualización, la práctica intelectual que proyecte e imponga categorías sobre la realidad, aun cuando utilice el léxico marxista, atenta contra cualquier análisis rigurosamente crítico e historicista. El problema es metodológico, pero tiene implicancias drásticas en el terreno político. La aplicación ciega de algunas categorías marxistas al estudio de la realidad argentina contemporánea por parte de los intelectuales del PCA -quienes no son mencionados directamente- es un insulto al propio pensamiento del intelectual alemán. Este planteo se observa en el artículo de Oscar Del Barco, Las formaciones económicas precapitalistas en Karl Marx, aparecido en el número 9 de PyP del año 1965:

El peligro radica en la tendencia a convertir estos esquemas en un a-priori del conocimiento histórico: en embretar los hechos en los esquemas y no, como es lo correcto en una metodología crítica, en usar los esquemas o modelos en su valor operatorio de intelección de los hechos 22 .

Al mismo tiempo, en el primer número de la revista también se observa la aparición de un modelo maniqueo que constituyó una constante en la revista: la dicotomía entre un socialismo "ortodoxo" y otro "crítico". Mientras el primero está identificado con un marxismo dogmático y economicista, el segundo término define al marxismo humanista, dentro del cual se inscriben los redactores de este colectivo cultural. Esta bifurcación se aprecia en el artículo de Del Barco "Carlos Marx y los manuscritos económico-filosóficos de 1844". En el mismo, el autor menciona la tendencia de ciertos sectores socialistas a escindir el trabajo intelectual de Marx, priorizando los textos económicos del Marx maduro por sobre sus trabajos juveniles. Este divorcio de la obra de Marx en etapas corrompe la totalidad de su trabajo, desvalorizando algunos problemas vitales de la sociedad contemporánea cómo la temática de la alienación. Esta preocupación por rescatar los escritos tempranos de Marx, centrándose en la categoría de alienación, también es una constante que vemos en La Rosa Blindada y las revistas del grupo comandado por Castillo.

A causa de este número inicial, devino la expulsión partidaria del comité redactor de PyP. En consecuencia, los números dos, tres y cuatro de dicha revista, publicados entre julio de 1963 y marzo de 1964, fueron escenario de una disputa que ya no aparece encubierta. Hacia el quinto número de la publicación, la disputa fue apaciguada, perdiendo intensidad e importancia. Expulsados del PCA, los

21 Marx Karl, El 18 Brumario de Luis Bonaparte, Buenos Aires, AGEBE, 2006.

22 Aricó José María, Pasado y Presente: edición facsimilar, Buenos Aires, Biblioteca Nacional, 2014. 
jóvenes cordobeses catalizaron una auténtica batalla de las ideas. A lo largo de sus artículos, se hace presente una argumentación común: tanto en su actualidad, como en múltiples acontecimientos de la historia contemporánea, PyP considera que el pensamiento dogmático fue ejecutor de una serie de derrotas en el proletariado mundial. Para ellos, esta crisis del pensamiento dogmático y "stalinista" es el resultado de la transformación del legado marxista en una colección de fórmulas rígidas, de "la ideologización de la filosofía de la praxis"23. La rutinización de viejas fórmulas - "la tradición de las generaciones muertas"- vuelve estéril las fuerzas vivas de la realidad social, es decir, el accionar esporádico pero frecuente- de las masas para derrumbar todo lo existente.

El grupo editorial tomó nota de los comentarios esgrimidos por sus adversarios políticos. Frente a la crítica de Rodolfo Ghioldi, líder emblemático del PCA, visible en la revista Nueva Era, la redacción no dudó en defender su posicionamiento. El postulado de Ghioldi es firme: el apartidismo es una claudicación frente a la burguesía y el accionar del grupo cordobés no es otra cosa que la eliminación de Lenin y su legado, a quien no osan mencionar a lo largo de sus páginas. La respuesta, también tajante, es un pedido por parte de la redacción de PyP para desacralizar los antiguos valores y mitos que están encubiertos detrás de las rígidas fórmulas y las frívolas frases repetidas hasta el hartazgo. Según el grupo pasadopresentista, la cantidad de veces que se cita a Lenin no es un criterio real para demostrar el grado de leninismo que pueda poseer una argumentación. Con respecto al líder soviético, existe una evidente disputa entre el PyP y el PCA para apropiarse de su figura. El grupo pasadopresentista criticó a su adversario intelectual a través del capital simbólico que representa la figura de Lenin, negándole su atribuido papel de defensores del marxismo-leninismo. En ese marco, el humanismo y la reivindicación de la praxis del sujeto están asimismo presentes:

Lenin representó en el movimiento revolucionario el desarrollo creador del pensamiento marxista en una nueva etapa histórica, la adecuación de los instrumentos organizativos del proletariado a las nuevas tareas revolucionarias, la revalorización de la subjetividad, de la actividad humana práctica, de la dialéctica como núcleo central del pensamiento marxista... El leninismo significó una nueva etapa de la unidad marxista-mundo real... No es por tanto una suma de principios que permanecen inmutables al margen de las realidades objetivas ${ }^{24}$.

De igual forma, el colectivo cultural agrupado en La Rosa Blindada formulará en los números pioneros de su emprendimiento algunas críticas sutiles al PCA. Su inicial preocupación rondará en torno a una reivindicación del realismo socialista como camino estético. Según la perspectiva del colectivo, el realismo socialista es una denominación que bajo ningún punto de vista debe ser asociada a la literatura realista producida exclusivamente por los soviéticos. Este criterio meramente estatal y nacional encubre la real potencialidad del género. Desplegado de su velo "dogmático" -nuevamente, aparece utilizado esté término de modo peyorativo-, esta corriente estética expresa un reflejo filosófico estructurado por una visión del mundo marxista y humanista. Con respecto a esto, comenta Brocato en su artículo "Defensa del realismo socialista" del tercer número del año 1964:

Ese miembro maligno... da normas; indica cómo se debe escribir, señala temas y también los destierra; legisla procedimientos; determina que parte de la realidad debe reflejarse y que reflejos artísticos denuncian una concepción antipartidista... ${ }^{25}$.

Para los integrantes de RB, la pretensión de imponer cánones y técnicas únicas, con la intencionalidad de excluir cualquier obra que atente contra los "delirios" artísticos del Estado soviético,

23 Idem.

24 Idem.

${ }^{25}$ Brocato Carlos Alberto \& Mangieri José Luis, La Rosa Blindada: edición Facsimilar, Buenos Aires, Biblioteca Nacional, 2014. 
destruiría la autonomía y personalidad del sujeto creador, elemento vital para la producción literaria. El monolitismo impuesto por la Unión Soviética hacia el interior de su territorio es un aspecto común en todos los partidos comunistas del mundo, dando por sentado, sin mención alguna, una actitud similar en el PCA. Para alimentar esta idea, en los números iniciales de la revista se publicaron varias entrevistas a Jean-Paul Sartre, en donde se encuentra reflejado el conflicto del intelectual con el partido comunista francés por la publicación de su obra de teatro "Las manos sucias"26.

Tras la expulsión de Brocato y Mangieri del PCA, la revista lanzó una serie de proclamas con la finalidad de discutir el papel del artista revolucionario y los fundamentos de una estética socialista. Nuevamente, observamos una apelación necesaria a la categoría de alienación, así como una defensa de los escritos juveniles y "hegelianos" de Marx. El acercamiento a dichos problemas se entiende en el marco de una vocación de la revista de problematizar la situación de la sociedad argentina contemporánea. La alienación será retomada en múltiples artículos, ninguno escrito por un miembro activo de RB: un trabajo de Antonio Caparrós sobre el incentivo material en el espacio laboral soviético, una biografía de la obra de Roger Garaudy por parte de Oscar Terán, un escrito de John William Cooke sobre las tentativas bases para una política cultural revolucionaria y una polémica de León Rozitchner, "La izquierda sin sujeto". Todos estos artículos recrean un dilema constante dentro de este colectivo cultural: el lugar del sujeto y de la praxis humana en el devenir revolucionario.

Desde otro matiz, la lucha del grupo de Abelardo Castillo contra sus adversarios comunistas se encuentra presente en los números iniciales de sus emprendimientos. Este colectivo nace de una separación explícita con la revista Gaceta Literaria, por lo que las diferencias no tendrán que contenerse obligatoriamente. Con un sentido ambiguo, oscilando entre un tono cómico y burlón y una crítica seria y detallada, los números iniciales de El Grillo de Papel denostarán un acento controversial contra sus antiguos compañeros. Surgido durante los meses finales del año 1959, EGDP lanzó ataques directos e indirectos a sus rivales. Al mismo tiempo que publica pésimas y peyorativas críticas contra la novela "Las hermanas" de Pedro Orgambide -director de Gaceta Literaria- y "Sonata popular de Buenos Aires" de Julio Huasi -editado por Cuadernos de Cultura, perteneciente al PCA-, la editorial que da apertura a la revista tuvo una tonalidad poco conciliadora. Preconizando la importancia del arte como un fin en sí mismo, pero que, adrede, es un utensilio fundamental para el cambio social, Castillo advirtió -refiriéndose al PCAque:

Para quienes prefieran el rótulo, la ordenada etiqueta sobre la frente, el "ismo" tranquilizador, confesamos que -en cuanto escritores- no nos parece imperioso llevar el carnet en el bolsillo, o distintivo de solapa, para escribir una gran novela, y -en cuanto hombres- tampoco creemos que esos sean elementos "sine que non" de la transformación social ${ }^{27}$.

Atribuyendo a la revista de Orgambide cierto apego a los perfiles estéticos soviéticos, el segundo número de EGDP divulgó un informe de Arnaldo Liberman sobre la situación del cine en el territorio socialista. En "Palabras para un cine polémico", Liberman cuestiona la intervención estatal en el arte a expensas de la libertad individual. Rememorando segmentos del pasado soviético, define al stalinismo como una primera instancia de control burocrático del arte ${ }^{28}$. La respuesta por parte de Gaceta Literaria no tardó en llegar. En su decimonoveno número, bajo el título de "Tzquierda y facilidad", Orgambide demostró un profundo malestar contra el colectivo de Castillo. Haciéndose ecos de las críticas, Castillo

\footnotetext{
${ }^{26}$ Haciendo eco de las ambigüedades entre compromiso e ideales políticos, la obra describe la vida de Hugo Barine, joven militante comunista que bajo el pedido de sus superiores asesina a un líder comunista acusado de traición.

27 Castillo Abelardo, El Grillo de Papel: edición facsimilar, Buenos Aires, Biblioteca Nacional, 2015.

${ }_{28}$ Comenta en dicho artículo Liberman: "En la primavera de 1930 surgió para el cine, la escultura, el teatro y la pintura soviética, un plan de funcionarios. Dicho plan admitía el control burocrático, la censura política, el dirigismo de la ortodoxia y hasta la prescripción de la técnica artística que debía usarse...”. Idem.
} 
dedica la editorial de la cuarta edición de EGDP a discutir con Orgambide. En ésta, el mismo aboga por el derecho de su revista a criticar la ortodoxia del PCA, así como toda práctica que, en sus consideraciones, atente contra la construcción revolucionaria. A su vez, enfatiza en la caracterización que Orgambide realizó sobre ellos como aparentes abogados del individualismo humanista. Sobre esa cuestión, nuestro protagonista equipara al socialismo con un auténtico humanismo, en donde la libertad personal y la revolución son dos actos unívocos. Citando brevemente varios escritos de Marx -los famosos Manuscritos económicos y filosóficos de 1844- y Engels, Castillo vinculó el proceso de liberación social con la defensa del individualismo del sujeto, aspecto que aparece simbióticamente relacionado con la libertad de expresión y obra.

La represión ejercida contra los grupos editoriales por el entonces gobierno de Arturo Frondizi mutó radicalmente el panorama y la línea política del grupo de Castillo. La acción gubernamental procedió a clausurar la imprenta Stilcograf, organismo dentro del cual se engendraba no solamente EGDP sino también, Gaceta Literaria. La medida catalizó un cambio de rumbo por parte del colectivo liderado por Castillo. En primer lugar, el órgano de expresión del grupo transmuta tomando como nueva identidad El Escarabajo de Oro. En ese marco, la consideración de esta nueva revista hacia la cultura comunista tiene una nueva dirección: de la confrontación directa, el colectivo pasa a hacer un intento de acercamiento concreto, aunque dicho objetivo no está libre de tensiones y conflictos. La política represiva de Frondizi -y la de sus sucesores- incitó al núcleo de EEDO a promover una unidad de acción literaria y artística, con la finalidad de unificar contra la política represiva a todo sector que se considere de izquierda. Esto se reflejó en la elaboración de comunicados comunes entre las revistas, la formación de listas en conjunto para las elecciones de la SADE (Sociedad Argentina de Escritores) y la invitación a viejos rivales como Pedro Orgambide a escribir en EEDO. ${ }^{29}$ No obstante, la voluntad de formar dichos espacios no llevó a una sumisión total frente a la estructura del PCA. Si bien con una tonalidad más abierta y con aires visiblemente más conciliatorios, manifestado en los comunicados comunes y el llamado a la unidad, las provocaciones de los dirigentes del partido comunista fueron frenéticamente rechazados. Dichas peleas y ataques siempre fueron colocadas en un contexto particular, de modo que cada crítica se realizaba sin olvidar la relevancia de generar una real unificación dentro de la izquierda. Esta dinámica aparece ilustrada en la descripción de un conflicto que EEDO entabló con Cuadernos de Cultura ${ }^{30}$ durante el año 1961. El segundo número de EEDO, divulgado en agosto de 1961, contiene la narración de una fricción con el PCA que ocupará varias ediciones de la revista, acompañada incluso por la difusión de un boletín especial sobre esta temática -publicada en separata-. La lucha inicia a partir de la publicación del número cincuenta de Cuadernos de Cultura, centro de divulgación del PCA, durante los inicios de ese mismo año. En dicho suplemento, la revista comunista difundió una serie de ensayos para debatir un tema vital para la historia política-cultural contemporánea: qué es la izquierda. Dos trabajos en particular, "La crisis del marxismo" de Héctor. P. Agosti y "Intelectuales y neo-izquierda" de Samuel Schneider, dedican su espacio a estigmatizar la orientación ideológica del grupo de Castillo -así como de muchos otros-. Acusando al colectivo editor de EGDP de intelectuales liberales y apartidarios, los ensayos de Cuadernos de Cultura apuntan al hecho de que estos emprendimientos independientes facilitan las tareas a la derecha política - "le hacen el juego"-. Con un estudio riguroso sobre el surgimiento de esta neo-izquierda, estos trabajos de los intelectuales comunistas intentan demostrar cómo estos colectivos culturales priorizarían sus intereses particulares por sobre la causa mayor de la emancipación proletaria, luchando contra los principios del marxismo.

Incapacitados temporalmente para responder a esa provocación por la clausura de la imprenta Stilcograf, las imputaciones a las afirmaciones de Agosti y Schneider se realizaron con la aparición de

\footnotetext{
${ }^{29}$ Esta apertura no impidió al grupo editor de EEDO criticar y elogiar simultáneamente, con ese tono cómico y sarcástico, la publicación de Hoy en la Cultura, nuevo emprendimiento de Orgambide.

30 Para un análisis histórico-político de dicha publicación, ver Cernadas, Jorge (2005). La `vieja izquierda`en la encrucijada: Cuadernos de Cultura y la política cultural del Partido Comunista argentino (1955-1963). X Jornadas Interescuelas/Departamentos de Historia. Escuela de Historia de la Facultad de Humanidades y Artes, Universidad Nacional del Rosario. Departamento de Historia de la Facultad de Ciencias de la Educación, Universidad Nacional del Litoral, Rosario.
} 
EEDO. Desde la consideración de Castillo, el dilema central de la izquierda es su incapacidad de diálogo entre pares. Mientras los sectores de la burguesía conocen donde está el enemigo revolucionario, la izquierda parece concentrada en disputas hacia su interior. Citando una célebre frase del popular libro Martín Fierro, Castillo apuesta por la unidad. Unidad que debe ser abierta a los sectores que no se encuentran representados en ningún partido. El compromiso de este colectivo es únicamente con la literatura y el arte; no se desestima la voluntad de un intelectual de intervenir mediante un partido, pero se desconoce a este accionar como una condición necesaria y obligatoria. Según Castillo, esa distancia con respecto a la estructura partidaria cobra más sentido en la contemporaneidad nacional, en donde ninguna organización y, muchos menos, el PCA, ha mostrado ser la cabeza directora del proletariado, ni en plano político ni, en menor grado, en el cultural. Adrede, la condena a la actividad del PCA toma un alto grado de importancia al instante de adentrarse en el pasado inmediato. Terco y "ortodoxo" frente a un fenómeno como el peronismo -al cual inicialmente, tampoco EEDO parece ver favorablemente- la falta de lucidez de los dirigentes comunistas abre paso a errores vergonzosos como su integración en la Unión Democrática de 1945. Posteriormente a este conflicto, la mención al PCA disminuye exponencialmente en la revista, la cual abre su horizonte hacia nuevos ejes temáticos.

En síntesis, los debates reconstruidos en la totalidad de los tres colectivos estudiados nos muestran dos elementos a tomar en consideración. Por un lado, existe un contexto espacial y temporal, ubicado entre la irrupción de la Revolución Cubana en 1959 y la llegada del Cordobazo en 1969, dentro del territorio argentino, en donde se observa la aparición de una nueva generación de intelectuales -en la mayoría de los casos, jóvenes- despegados de los ideales que hasta ese entonces definían a los pensadores de la izquierda política. Por el otro lado, y ligado al punto anterior, estos sectores utilizarán el conflicto y la disputa con su antiguo corpus teórico - en este caso, comunista- como un medio necesario tanto para poder desprenderse de esa tradición como para poder definir una nueva identidad y perspectiva política. Tras ese proceso de distanciamiento mediante algunos altercados, muchas veces iniciados por las provocaciones de sus viejos colegas, estos intelectuales quitarán importancia a las querellas con el comunismo nacional para poder abordar nuevos problemas culturales. En definitiva, la disputa con el PCA no tenía como objetivo central producir un efecto drástico en el campo político-cultural -aunque finalmente lo hizo-, sino más bien, fue un accionar que permitió a estos grupos unificar posiciones y afianzar una identidad singular ajena a la comunidad intelectual de donde procedían.

\section{Tiempo revolucionario y condiciones para la liberación: el por qué no de un partido}

La formación de una revista implica la cristalización de un deseo común entre un conjunto diverso de voluntades individuales. Sin embargo, la existencia de un objetivo universal no excluye la existencia de diferencias y disputas, a veces silenciosamente veladas, dentro de los integrantes de estos colectivos. En los casos estudiados en este artículo, la unificación inicial parece radicar en la necesidad de encontrar un nuevo espacio político, identificado dentro de la mentalidad de izquierda, el cual no esté sometido a los vaivenes políticos e intelectuales de una organización partidaria como el PCA. Pero esta aglutinación inicial puede desestabilizarse al momento de expresarse ciertos problemas para los cuales no existían previamente consenso. La corta duración de muchos de estos emprendimientos culturales puede comprenderse en parte por la existencia de condiciones externas a dichas publicaciones. Generalmente, la finalización de una revista se puede dar por una acción coercitiva -como vimos en el caso de EGDP, por la política represiva de gobierno de Frondizi- o, más normal aún, por la insuficiencia de medios económicos para sostener la edición de la misma. No obstante, la destrucción de estos colectivos no es producto únicamente de elementos externos. Más bien, el carácter efímero de una revista política-cultural se explica por una combinación entre condicionamientos acontecimentales-estructurales y los conflictos de las subjetividades particulares de quienes conforman dicha publicación. En ese marco, situaciones 
como el cambio de una coyuntura política o la aparición de una disputa intelectual que apele a los integrantes de la revista puede promover la apertura a nuevas tensiones dentro del colectivo, las cuales vienen a desmembrar los elementos comunes que habían permitido originalmente la creación del grupo. Este fenómeno toma más relevancia si la revista tiene un perfil que aluda inmediatamente a la praxis social, en la medida que una revista de vanguardia está más profundamente unida a las oscilaciones de la política. Estos problemas no serán abocados aquí por una cuestión de espacio. Lo central en esta situación es comprender que los colectivos culturales analizados en este trabajo son espacios que en menor o mayor grado se consideran como órganos que accionan en el terreno de lo político. Cómo fue indicado al inicio de este escrito, una pregunta legítima sería plantearse por qué estos grupos, heréticos con respecto a la cultura comunista a la cual pertenecían -orgánicamente o no-, no consideraron como una vía concreta la creación de una organización partidaria alternativa. El presente segmento apuntará a crear algunas posibles explicaciones a esta cuestión.

Inicialmente, cabe señalar que la creación de revistas como terrenos de disidencia es una tradición propia de la intelectualidad argentina, cuyas experiencias se remontan a los comienzos de la década de 1950. La lógica parricida de la juventud crítica nacional, agrupada en torno a la redacción de una revista, toma una instancia inicial en la necesidad de discutir abiertamente la cultura argentina luego del golpe de Estado de 1955 al gobierno de Juan Domingo Perón. Siendo los emprendimientos de Contorno (19541959), Ciudad (1955-1956) y Centro (1951-1959) algunos de sus ejemplos más paradigmáticos, la juventud congregada en estas revistas tenía como característica una mayor sensibilidad por lo nacional como temática y problema. De este modo, la constitución de una revista como una forma de expresión herética es un elemento con una temporalidad muy anterior a la formación de la nueva izquierda argentina, sin originarse, además, en el propio campo comunista. Sin embargo, esta usanza de los pensadores críticos nacionales no es explicativa en sí misma. Las causas por las cuales estos colectivos no devinieron en partido deben buscarse en las propias identidades y discursos de las revistas, cuyo folklore es completamente diferente entre sí.

Los tres colectivos culturales compartían una creencia común: el PCA no solo tenía un pensamiento y modus operandi arcaico, sino que, además, no tenía conciencia sobre la relevancia del trabajo intelectual y cultural en el plano pragmático. Sumido en las antiguas epopeyas y los hábitos partidarios, el comunismo argentino negaba toda novedad que se aleje de la "ortodoxia" stalinista. Configurado en un sentido de la historia lineal, etapista y secuencial, el PCA se adscribía a un modelo revolucionario único: el mito de la Revolución Rusa de 1917. No obstante, los discursos de las revistas analizadas hacían énfasis en despojar de un carácter teleológico al momento revolucionario, marcando un amplio complejo de posibilidades y caminos emancipatorios. De hecho, la Revolución Rusa de 1917 como práctica desviada ${ }^{31}$ suponía el acontecimiento universal que destroza, en los sucesos concretos, las teorías que formulan esquemas rígidos de trasformación social:

El complejo de posibilidades revolucionarias abierto por las masas que en 1917 conquistaron el poder en Rusia... asume hoy una riqueza inesperada. Las posibilidades de los caminos revolucionarios son tan amplios y diversificados que la teoría amenaza quedar permanentemente rezagada y hasta "anacrónica"32.

Para estos grupos de intelectuales, el mapa y teorema revolucionario al que adscribía el PCA era caduco frente a la situación contemporánea nacional y mundial. Alejados de una perspectiva etapista, los relatos de nuestros tres colectivos culturales poseían una visión abierta y multi-temporal de la historia.

\footnotetext{
31 En gran parte de la tradición marxista y socialista clásica, Rusia no era considerada un territorio en cuyos límites se reúnan las condiciones para el proceso revolucionario. Atrasada y primordialmente agraria, Rusia debía esperar la próxima industrialización venidera. Según estas visiones, asimilada Rusia a los países europeos avanzados a partir de una revolución burguesa, el naciente proletariado tendría la conciencia y grado suficiente para desplegar la fase socialista de la liberación.

32 Aricó, op. Cit.
} 
Reflejo de tal visión se observa en el comentario que Héctor Schmucler redacta en su artículo Problemas del Tercer Mundo, del cuarto número de PyP del año 1964:

Si la revolución es una elección humana a partir de condiciones materiales determinadas, los caminos de la misma pueden ser múltiples...Pretender recetas generales (sean habladas en ruso o en chino) es ignorar que la revolución expresa una voluntad originaria. Es la metafísica de la revolución ${ }^{33}$.

Tales planteos también aparecen presentes en diversas advertencias mencionadas en los artículos de RB. Ya sea en los textos de Brocato sobre el realismo socialista o en las colaboraciones teóricas de León Rozitchner, nuestros pensadores previenen sobre los peligros y sinsentidos de utilizar ciertas categorías históricas, naturalizadas dentro de la mentalidad comunista, que tracen como inevitable la llegada del socialismo, como es la situación de las ideas del "optimismo histórico" o "leyes de la dialéctica", entre otras. En su artículo La izquierda sin sujeto, Rozitchner señala:

La rigidez no es un atributo sólo de la derecha, así como el realismo no es una virtud que convenga siempre a la izquierda. Es fácil verificarlo: los que están a la izquierda -muchos de ellos- se complacen en hablar de las "leyes de la dialéctica", de las "leyes del desarrollo económico", de las "leyes de la lucha de clases" y de la "necesidad histórica de la revolución”, todo lo cual encuentra su término en una certeza final: el necesario tránsito del capitalismo al socialismo ${ }^{34}$.

En ese sentido, la revolución no era considerada como un proceso diagramado, un programa diacrónico cuyas instancias están inscriptas en el desarrollo social. El tiempo revolucionario, como momento de accionar de los sublevados, era percibido como una instancia que habilita la intersección de una amalgama de tiempos, donde se conectaba un pasado casi olvidado, un presente intenso y un futuro potencialmente manumitido. Bajo ese ángulo, aparece visible en ciertos pasajes del discurso de nuestras revistas, de manera sutil, como breves y acotados respiros, un sentido histórico que se asimila a la categoría benjaminiana de tiempo-ahora. Entendido como una temporalidad que destruye el continum de la historia, el tiempo-ahora refiere a un momento, a un instante fugaz, en donde se condensa y comprime diversas temporalidades, acontecimientos y lecturas del tiempo: una situación donde se resume abreviadamente la historia de la humanidad como totalidad. El tiempo-ahora alude a una situación en donde confluyen diversas experiencias sociales marcando un lazo directo entre pasado, presente y futuro -multitemporalidad-. Este proceso apunta a la remembranza, aspecto que implica el apoderamiento y la actualización de un recuerdo pasado. Como modelo del tiempo mesiánico, el tiempo-ahora simboliza un salto de tigre hacia el pasado gestado por las clases oprimidas: un acto dialéctico y explosivo, que enzarza la conciencia por parte de esos actores, en ese instante de acción, de la ligazón de dicho escenario actual con la experiencia del pasado ${ }^{35}$. Estas afirmaciones no implican negar la existencia de ciertas percepciones temporales lineales o hegemónicas dentro de los discursos de nuestras revistas. No obstante, ello no impide observar una tratativa singular de estos grupos intelectuales para hablar de lo que ellos consideraban el proceso de emancipación social. La descripción de las experiencias revolucionarias pasadas como aristas que, despojadas de toda esquematización, pueden ser una herramienta retomada en el presente para un futuro liberado, es un elemento sustancial en nuestros colectivos culturales:

La historia del movimiento revolucionario es lo suficientemente rica como para que pretendamos olvidarnos de sus enseñanzas. No es casual que sea precisamente en los

\footnotetext{
33 Idem.

34 Brocato \& Mangieri, op. Cit.

35 Löwy Michael, Walter Benjamin. Aviso de incendio: una lectura de las tesis "Sobre el concepto de historia". Buenos Aires, Fondo de Cultura Económica, 2012.
} 
momentos de apertura de las "situaciones revolucionarias", en las urgencias que crean los grandes cambios históricos, cuando los marxistas deben mostrar su condición de tales. En ese sentido, nunca fue Lenin más filósofo que en el periodo que precedió a la revolución de Octubre ${ }^{36}$.

La interrupción mesiánica tiene un contenido particular: el mesías es redentor. El objeto de la revolución en Benjamin es visibilizar y vengar a los muertos derrotados y vencidos del pasado, que aparecen ocultados por la historia de la clase dominante. En este punto, nuestras revistas toman una visión diferente: su mirada está, contrariamente, colocada hacia el futuro. Su objetivo no era la redención de los mártires, sino la liberación de la Argentina y la humanidad. De esta forma, la revolución de 1917 emerge conectada con toda una serie de sucesos de emancipación social, cuya expresión actual era claramente la Revolución Cubana y las rebeliones del Tercer Mundo:

Cuba, lo comprendíamos entonces... significa la empedrara historia de la lucha por la dignidad del hombre, por su libertad, por su decencia, por su última y definitiva belleza, un acontecimiento tan luminoso para América Latina como lo fue, para el socialismo del mundo, la revolución de $1917^{37}$.

Supuestamente, somos revolucionarios; evocamos pues, con ferocidad, palabras de Marx, sobre que no se trata de hacer cambiar de mano la maquinaria burocráticomilitar, etcétera...Pero, al mismo tiempo, nada nos impide acordar, sin demasiado escándalo, que las condiciones históricas de nuestro país - hic et nunc- no son las de Cuba hace cinco años, claro; ni las de Rusia hace medio siglo ${ }^{38}$.

En ese sentido, una primera explicación para el no desarrollo de una organización partidaria puede radicar en este rechazo rotundo a ese espacio organizativo del cual procedían la mayoría de estos jóvenes intelectuales. Frente a un mundo -un Tercer Mundo- extremadamente convulsionado, las fuerzas vivas de los movimientos sociales opacaban al difunto partido político. No obstante, los discursos revisteriles no tenían un posicionamiento cerrado respecto al partido político. Si bien no lo reconocían como el único camino revolucionario, los tres colectivos consideraban que es un mecanismo viable y que, en ciertas condiciones, podía ser el más indicado para la transformación social. Consecuentemente, el problema residía en que la caracterización de la situación política actual no amerita la formación de un partido. Por consiguiente, en su consideración el partido no era la vía adecuada, en la medida que no existían disposiciones -sociales y culturales- indicadas para su desenvolvimiento. Adrede, sus circunstancias actuales colocaban a las revistas y a la actividad cultural como un medio -parcial, pero efectivo- para actuar dentro de la mentalidad de la población nacional. Esta concepción aparecía más presente en el grupo de Abelardo Castillo, el cual reiteradamente planteaba como prioridad la literatura como un fin en sí mismo. De esa afinidad por el arte resultaba su responsabilidad política, cuya praxis deviene en un compromiso intelectual tipo sartreano. Por otro lado, la diversidad de juicios políticos existentes dentro del grupo hubiera, además, tornado extremadamente dificultoso la posibilidad de poner en funcionamiento un partido político. En palabras de Liliana Heker, una revista no es un simple órgano de expresión de corte político, sino que implica la articulación de ese aspecto con la vitalidad de los intereses cambiantes de sus redactores, los cuales pese a los centelleos de sus deseos siempre expresan una esencia propia y común:

Una revista es, no, como lo pretendió Cuadernos de Cultura, un "programa": una cosidad estática y durísima sino un organismo que se va configurando, modificando dialécticamente y naciéndose a cada muerte, a través de su historia; y que se juzga

36 Aricó, op. Cit.

${ }^{37}$ Castillo Abelardo, El Escarabajo de Oro: edición facsimilar, Buenos Aires, Biblioteca Nacional, 2015

${ }^{38}$ Idem. 
como totalidad: a lo largo y a lo hondo. Por la obra total, cuando acaba, y por praxis mientras vive. Y la praxis de un grupo de escritores es, también, la literatura. Pero, de literatura, nunca hablaron nuestros críticos ${ }^{39}$.

Un escenario similar puede formularse para el colectivo nucleado en torno a RB. Concebida como una publicación de estética, la politización de la revista adquirió mayor grado de relevancia con la expulsión de Mangieri y Brocato del PCA. Sus fundamentos y principios eran otros, detallando que su lugar como pensadores se definía, en última instancia, como ese "compañero de ruta" partidario del que tanta gala hacia Jean-Paul Sartre. Eventualmente, tanto los miembros de RB y PyP tendrían una seria dificultad práctica para formar una ruptura partidaria. Como cuadros intelectuales menores o integrantes ligados al espacio cultural del PCA, estos colectivos no tendrían artilugios militantes ni políticos para formar un partido o fracción propia. Su poco peso dentro de un partido de pocas dimensiones hubiera limitado su capacidad de "ganar" a parte de la militancia para su potencial organización. Sumado a ello, su nula presencia en espacios sindicales o sociales hacía imposible cumplir con una de las facultades elementales de una revista, según su propio criterio: como comenta Aricó, “...las revistas pueden cumplir con esta verdadera acción de organización de la cultura solo en cuanto devienen centros de elaboración y homogeneización de la ideología de un bloque histórico en el que la vinculación entre la elite y masa sea orgánica" 40 .

Conjuntamente, no hay registros de un eventual accionar de estos colectivos con la intención de poner en funcionamiento una posible organización partidaria. Esa cuestión, en estrecha vinculación a los discursos presentes dentro de estos artefactos culturales, nos permite considerar que, a grandes rasgos, la voluntad de gestar una revista como expresión de la disidencia en lugar de un partido se debe a un aspecto más programático que circunstancial. Más allá de la posible ausencia de condiciones para formar una agrupación política, es la propia personalidad de estos intelectuales lo que promueve esta dirección elegida. Siguiendo las perspectivas detalladas por Perry Anderson, podemos concluir que los tres colectivos estudiados no procedieron a mantener una unión orgánica entre teoría y praxis, acción propia de los pensadores clásicos del marxismo. Optando por perpetuar el divorcio estructural entre teoría y práctica política, la identidad de nuestros grupos puede asimilarse estrechamente con la tradición propia del marxismo occidental ${ }^{41}$.

\section{Palabras finales}

Si el derrotero del comunismo internacional se data temporalmente a inicios de la década de 1990, este articulo da muestras de que existían previamente muchos resquebrajamientos de esta tradición. Si bien a partir de la muerte de Stalin las disidencias se harán más explícitas, lo cierto es que el pensamiento comunista es un corpus teórico y político en constante tensión y conflicto, el cual está en permanente destrucción y reconstrucción -incluso hasta hoy en día-. El monolitismo con el que nuestros colectivos intelectuales analizados definían a su adversario comunista puede tener cierta base argumental real. Sin embargo, este adjetivo oculta el hecho de que al interior de esa aparente rigidez se dejaba entrever ciertos aires de cambio, de los cuales nuestras cuatro revistas son un caso paradigmático.

Del estudio realizado podemos concluir varias cuestiones. Centralmente, las discusiones que PyP, RB, EGDP y EEDO emprenden contra el PCA o sus artefactos culturales se inscriben dentro de la eterna querella del marxismo, pero, a su vez, implican también un mecanismo que los permitió afianzarse como colectivos y otorgarles una marcada identidad singular. La reivindicación del marxismo humanista es el parámetro primordial para poder aglutinar a todas estas revistas, además del evidente hecho de que todas

\footnotetext{
${ }^{39} \mathrm{Idem}$.

40 Aricó, op. Cit.

${ }^{41}$ Anderson Perry, Consideraciones sobre el marxismo occidental. Séptima edición, Madrid, Siglo veintiuno editores, 1987.
} 
Francisco Caamaño, Discípulos y disidentes: la formación de las revistas político-culturales como espacios de ruptura frente al

Partido Comunista argentino (1959-1969), Izquierdas, 49, julio 2020:1018-1035

estas experiencias tuvieron un lazo previo con la tradición comunista. En sintonía, la creación de revistas como forma de expresar la disidencia se explica por la conjunción de una serie de factores, dentro de los que se puede mencionar la herencia intelectual de estos jóvenes, su nulo capital político o incapacidad de arrastrar consigo a una base de apoyo político -ya sea del PCA o nueva- y las orientaciones ideológicas de estos grupos. Pero, primordialmente, podemos afirmar que nuestros protagonistas partían de una base conceptual en la cual la estructura partidaria no es un elemento imprescindible para el proceso revolucionario. En la medida en que ellos denigraban una visión evolutiva, gradual y esquemática de la transformación social en pos de una conceptualización disruptiva, multilineal y ligada a saltos históricos -que en algunos sentidos se asemeja a la idea del tiempo-ahora mesiánico de Walter Benjamin-, la creación del partido estaba lejos de ser, en su consideración, una tarea inmediata para un colectivo revolucionario. En este sentido, este trabajo intenta aportar, tal vez sin éxito alguno, un conjunto de nuevas aristas para poder problematizar el nacimiento de la nueva izquierda intelectual en la Argentina.

\section{Bibliografía}

Anderson Perry, Consideraciones sobre el marxismo occidental. Séptima edición, Madrid, Siglo veintiuno editores, 1987.

Beigel Fernanda, Las revistas culturales como documentos de la historia latinoamericana, En Utopía y Praxis Latinoamericana, enero-marzo 2003, 105-115.

Campione Daniel, El Partido Comunista de la Argentina. Apuntes sobre su trayectoria, Coloquio internacional: El comunismo. Otras miradas desde América Latina, México D.F, 2007.

Camarero Hernán, Tiempos rojos. El impacto de la Revolución Rusa en la Argentina, Buenos Aires, Sudamericana, 2017.

Cernadas Jorge, Pittaluga Roberto \& Tarcus Horacio, La historiografía sobre el Partido Comunista de la Argentina: Un estado de la cuestión, En El Rodaballo. Revista de política y cultura, 8, 1998.

Cuevas Agustín, El marxismo latinoamericano: historia y problemas actuales. En Entre la ira y la esperanza y otros ensayos de crítica latinoamericana, Bogotá, Siglo del Hombre Editores y CLACSO, 2008, 9-67. Falcón Ricardo \& Quiroga Hugo, Contribución al estudio de la evolución ideológica del PCA, En Contribuciones programa CLACSO-Santiago de Chile, 50, 1987.

Gilman Claudia, Entre la pluma y el fusil. Debates y dilemas del escritor revolucionario en América Latina, Buenos Aires, Siglo XXI, 2003.

Grande Cobián, Leonardo J., El programa político de El Grillo de Papely El Escarabajo de Oro (1959-1964), IV Jornadas de Sociología de la UNLP, La Plata, 2005.

Katchadjian Guillermo, La Rosa Blindada: entre la cultura de la rebelión y la política revolucionaria, IV Jornadas de Jóvenes Investigadores. Instituto de Investigaciones Gino Germani, Facultad de Ciencias Sociales, Universidad de Buenos Aires, Buenos Aires, 2007

Kohan Néstor, Cultura y revolución en La Rosa Blindada, Buenos Aires, Amauta Insurgente ediciones, 2015 Kohan Néstor, La Rosa Blindada: una pasión de los sesentas, Buenos Aires, La Rosa Blindada, 1999.

Löwy Michael, W alter Benjamin. Aviso de incendio: una lectura de las tesis "Sobre el concepto de bistoria". Buenos Aires, Fondo de Cultura Económica, 2012.

Massholder Alexia, El surgimiento de la revista Pasado y Presente. Una mirada desde lo generacional, Jornadas Internacionales José María Aricó, Córdoba, 2011

Marx Karl, El 18 Brumario de Luis Bonaparte, Buenos Aires, AGEBE, 2006.

Nállim, Jorge A., Intelectuales y Guerra Fría: El Congreso por la Libertad de la Cultura en Argentina y Chile, 1950-1964. En Anuario del Instituto de Historia Argentina, 14, 2014.

Petra Adriana, Intelectuales y cultura comunista: Itinerarios, problemas y debates en la Argentina de posguerra, Buenos Aires, Fondo de cultura económica, 2017.

Petra Adriana, Pasado y Presente: Marxismo y modernización cultural en la Argentina postperonista, En Historia y espacio, 41, 2013. 
Sigal Silvia, Intelectuales y poder en la década del setenta, Buenos Aires, Puntosur editores, 1991.

Siskindovich Santiago, El Partido Comunista Revolucionario y la construcción de la delimitación con el Partido Comunista argentino (1968-1969), En Izquierdas, 49, enero 2020, $43-57$.

Tarcus Horacio, Marx en Argentina. Sus primeros lectores obreros, intelectuales y cientificos, Buenos Aires, Siglo XXI, 2007.

Terán Oscar, Nuestros años sesenta. La formación de la nueva irquierda intelectual argentina (1956-1966), Buenos Aires, Puntosur editores, 1991.

Tortti María Cristina, El "viejo" Partido Socialista y los orígenes de la "nueva" izquierda (1955-1965), Buenos Aires, Prometeo libros, 2009.

Tortti María Cristina, Protesta social y "Nueva Izquierda" en la Argentina del "Gran Acuerdo Nacional", Taller. Revista de Sociedad, Cultura y Política, 6, 1998.

\section{Fuentes documentales}

Aricó José María, Pasado y Presente: edición facsimilar, Buenos Aires, Biblioteca Nacional, 2014.

Brocato Carlos Alberto \& Mangieri José Luis, La Rosa Blindada: edición facsimilar, Buenos Aires, Biblioteca Nacional, 2014.

Castillo Abelardo, El Escarabajo de Oro: edición facsimilar, Buenos Aires, Biblioteca Nacional, 2015. Castillo Abelardo, El Grillo de Papel: edición facsimilar, Buenos Aires, Biblioteca Nacional, 2015. 